\title{
Connection between the child's behavior in the dental office and premature tooth extraction
}

\author{
Radosveta Andreeva ${ }^{1}$, Milena Georgieva ${ }^{1}$, Evgeni \\ Dimitrov $^{1}$, Ani Belcheva ${ }^{2}$.
}

1. Department of pediatric dentistry, Medical University of Varna;

2. Department of pediatric dentistry, Medical University of Plovdiv;

\begin{abstract}
Dental anxiety and negative behavior are common problems in children. The aim of this study is to evaluate the connection between a child's behaviour in the dental office and premature tooth loss. Methods and materials: Subject of monitoring of the clinical research were 140 school children with mixed dentition. The clinical group consists of 90 children with prematurely extracted teeth. The patients from the clinical group were divided into three groups of 30 patients. The control group consists of 50 children with intact denture. The child's behavior was assessed by the denti using a behavioral scale. A special evaluation form for behavior assessment about the level of negativism of the children was filled by the examining dentist. Frankl's rating scale was used. This scale consists of four stages and each defines specific behavior. Results: There were no definitely negative children in our study. The results show that most of the patients from the control group are positive towards the dental treatment $-61.2 \%$ and only $14.3 \%$ are negative. About $35 \%$ of the children with prematurely extracted teeth are positive, most of them $-61.3 \%$ are negative. Conclusion: Most of the children with prematurely extracted teeth are negative towards dental treatment due to which they are with poor dental health and early tooth loss. The use of behavioural techniques are recommended in order to improve their oral health and to reduce the number of premature extractions.
\end{abstract}

Keywords: premature extraction, child's behavior, Frankl's scale, primary teeth 


\section{Introduction}

A child's behavior in the dental office is a fundamental concern in pediatric dentistry. The treatment of a local dental problem is not a matter of decision, the rule is that we need to heal and ingratiate the patient, especially when we talk about children - patients (1). Dental avoidance is a high risk factor for the development of odontophobia, negative behavior in the dental office and poor oral health including premature tooth extraction in children. The negative child's behavior presents a problem for the treatment of patients suffering from it, and also for the dentist $(2,3)$. A high level of dental negativism can influence the dental treatment in such a way that it becomes almost impossible for the dentist to complete the treatment $(3,4)$. A lot of research has shown that dental anxiety and negative behavior are one of the reasons for not seeking dental treatment $(2,5,6,7,8)$. A negative attitude towards dental treatment, because of fear, is often considered to be an essential emotion. It is therefore normal for little patients to be afraid of new and potentially threatening situations such as pulpitis and periodontitis treatment. It often happens that in such situations parents prefer to delay the treatment and this leads to premature tooth extraction in children $(9,10,11)$.

\section{Aim}

The aim of this study is to evaluate the connection between a child's behavior in the dental office and premature tooth loss.

\section{Material and Methods}

Subject to monitoring in the clinical research were 140 school children with mixed dentition. The clinical group consists of 90 children with prematurely extracted teeth. The patients from the clinical group were divided into three groups of 30 patients.

- Patients that have lost one or more tooth extracted in the dental clinic or that come to the clinic during the first 2 months after the extraction.

- Patients with premature temporary teeth extraction 6 months before they come to the dental office.

- $\quad$ Patients with premature temporary tooth extraction more than one year before they come to the dental office.

The control group consists of 50 children with intact denture. The children from the control group are the same age as the children from the clinical groups.

The child's behavior was assessed by the dentist using a behavioral scale.

A special evaluation form for behavior assessment about the level of negativism of the children was filled by the examining dentist. Frankl's rating scale was used. This scale consists of four stages and each defines specific behavior.

Stage 1 - definitely negative. The children refuse treatment, cry, and experience great fear.

Stage 2- negative. They are resistant to treatment, hostile, and shy. 
Stage 3- positive. These children accept treatment, but they are cautious and reserved, but follow the instructions of the dentist.

Stage 4 - definitely positive. The children demonstrate understanding. They are interested in dental procedures. This behavioral scale is one of the most commonly used ones for behavioral assessment and it is applied successfully in clinical practice.

\section{Results}

There were no definitely negative children in our study. The results show that most of the patients from the control group are positive towards the dental treatment- $61.2 \%$ and only $14.3 \%$ are negative. About $35 \%$ of the children with prematurely extracted teeth are positive, most of them $-61.3 \%$ are negative (Table 1 ).

\begin{tabular}{|c|c|c|c|c|c|c|c|c|}
\hline \multirow{3}{*}{ Groups } & \multicolumn{6}{|c|}{ Evaluation of the child's behavior } & \multirow{2}{*}{\multicolumn{2}{|c|}{ Total }} \\
\hline & \multicolumn{2}{|c|}{ Definitely positive } & \multicolumn{2}{|c|}{ Positive } & \multicolumn{2}{|c|}{ Negative } & & \\
\hline & Number & $\%$ & Number & $\%$ & Number & $\%$ & Number & $\%$ \\
\hline Control group & 12 & 24.5 & 30 & 61.2 & 7 & 14.3 & 49 & 100 \\
\hline Group 1 & 3 & 10.3 & 12 & 41.4 & 14 & 48.3 & 29 & 100 \\
\hline Group 2 & 0 & 0.0 & 11 & 36.7 & 19 & 63.3 & 30 & 100 \\
\hline Group 3 & 0 & 0.0 & 8 & 27.6 & 21 & 72.4 & 29 & 100 \\
\hline Statistical significance & & $x^{2}=3$ & $69, \mathrm{df}=6$ & $0<0.0$ & & & & \\
\hline
\end{tabular}

\section{Discussion}

The statistically significant correlation $(p<0.0001)$ can be explained by the unpleasant associations that the surveyed children make with dental treatment. Their visits to the dental office are associated with pain, caused by the manipulation of premature extraction or putting anesthesia. Some of them have a negative previous experience in the dental office. 
The manipulations that the children in the control group have been subject to are more common prophylactic, micro invasive and painless. Depending on the child's behavior in the dental office, when the patients are negative, the dentists must find an appropriate method to influence their behavior and predispose them to maximum assistance during treatment. The use of behavioural techniques is recommended to influence the negative behavior of children in order to reduce their fear of dental treatment $(12,13,14,15)$.

\section{Conclusion}

Most of the children with prematurely extracted teeth are negative towards dental treatment due to which they are with poor dental health and early tooth loss. The use of behavioural techniques are recommended in order to improve their oral health and to reduce the number of premature extractions.

\section{References}

1. Ilieva E. The child as a dental patient. PhD thesis Sofia 2000.

2. Smith TA, Heaton LJ. Fear of dental care: are we making any progress?. J Am Dent Asso. 2003;134(8):1101-1108.

3. Folayan MO, Idehen EE, Ojo OO. The modulating effect of culture on the expression of dental anxiety in children: a literature review. Int J Paediatr Dent. 2004;14(4):241-245.

4. Armfield JM, Spencer AJ, Stewart JF. Dental fear in Australia: Who's afraid of the dentist?. Aust Dent J. 2006;51(1):78-85.

5. Schuller AA, Willumsen T, Holst D. Are there differences in oral health and oral health behavior between individuals with high and low dental fear?. Community Dent Oral Epidemiol. 2003;31(2):116-121.

6. Sohn W, Ismail Al. Regular dental visits and dental anxiety in an adult dentate population. J Am Dent Assoc. 2005;136(1):58-66.

7. Long N. Stress and economic hardship: The impact on children and parents. Pediatr Dent 2014;36(2):109-14.

8. Boyce TW. The lifelong effects of early childhood adver-sity and toxic stress. Pediatr Dent 2014;36(2):102-7.

9. Moore R, Brodsgaard I, Rosenberg N. The contribution of embarrassment to phobic dental anxiety: a qualitative research study. BMC Psychiatry. 2004;4(1):10-11.

10. Frankel MR, Frankel LR. Some recent developments in sample survey design. J Market Res. 1977;14:280-293. 
11. Wells M, McTigue DJ, Casamassimo PS, Adair S. Gender shifts and effects on behavior guidance. Pediatr Dent 2014;36(2):138-144.

12. Armfield, JM, \& Heaton, LJ. (2013). "Management of fear and anxiety in the dental clinic: a review." Australian Dental Journal, 58(4), 390-407.

13. Potter, C., Kinner, D., Tellez, M., Ismail, A, \& Heimberg, R. (2014). "Clinical implications of panic symptoms in dental phobia." Journal of Anxiety Disorders, 28, 724-730.

14. American Academy of Pediatric Dentistry. Guideline on oral health care for the pregnant adolescent. Pediatr Dent 2015;37(special issue):159-165

15. Goleman J. Cultural factors affecting behavior guidance and family compliance. Pediatr Dent 2014;36(2):121-127.

\section{Corresponding author:}

Radosveta Andreeva

Faculty of Dental Medicine, Medical University of Varna 55 Marin Drinov Str. 9002 Varna, Bulgaria

Tel.:0887202 495

e-mail: doctor_ra@abv.bg 